\title{
Analysis of Long-Term Outcomes Following Surgical Contracture Release of the Elbow: A Case Series \\ Brittany M. Ammerman ${ }^{1}$, Gary Updegrove ${ }^{1}$, Padmavathi Ponnuru ${ }^{1}$, April Armstrong ${ }^{1}$ \\ 1. Orthopaedics, Penn State Health Milton S. Hershey Medical Center, Hershey, USA
}

Corresponding author: Brittany M. Ammerman, bammerman@pennstatehealth.psu.edu

Abstract
Background
Elbow contracture is a debilitating condition with an incidence ranging from as low as almost 1\% to as high
as $20 \%$ and results in significant limiting consequences on a patient's activities of daily living (ADLs).
Postoperative rehabilitation is important in maintaining the range of motion and sustaining an improved
range of motion. The purpose of this study was to evaluate the long-term results of elbow contracture
release surgery and the effect of an occupational therapy/physical therapy (OT/PT)-guided, self-directed
rehabilitation program following surgery, without the use of continuous passive motion (CPM) devices.

\section{Methods}

We enrolled patients who had undergone elbow contracture release surgery from 2005 to 2016 at a single institution under the senior author. The evaluation included objective measurements of range-of-motion, strength, and neurological sensory testing. Provocative testing of the elbow and hand was performed. American Shoulder and Elbow Surgeons-elbow (ASES-e), Simple Shoulder Test-elbow (SST-e), Disabilities of the Arm and Shoulder (DASH), Mayo Elbow Performance Index (MEPI), Short Form-36 (SF-36), and an investigator questionnaire were completed.

\section{Results}

We enrolled 19 patients, six female and 13 male, with an average follow-up of 58.9 months (SD \pm 39.8 , Range $22-117)$. We showed improvement and sustained motion between preoperative and postoperative research visit flexion $(\mathrm{p}<0.001)$ and flexion extension-arc $(\mathrm{p}<0.01)$. The mean increase in flexion was $98^{\circ}$ to $131^{\circ}$ and the flexion-extension arc was $36^{\circ}$. Patients were satisfied with the decision to undergo surgery and had sustained ability to complete ADLs.

\section{Discussion}

This patient cohort demonstrated a statistically significant increase, as well as long-term maintenance in the flexion and flexion-extension arc. A self-directed, OT/PT-guided, therapy program without CPM was effective. Patients showed good outcomes and were satisfied with their ability to perform ADLs, decreased pain, and the decision to undergo surgery.

\section{Introduction}

Elbow contracture is a debilitating condition with significant limiting consequences on a patient's activities of daily living (ADLs) [1]. Due to the elbow joint's distinct anatomy, particularly the presence of three separate articulations within a single synovial cavity, the abundance of soft tissue providing joint stability, and the proximity of the brachialis to the anterior capsule, the joint is susceptible to stiffness and contracture [2]. The range of motion (ROM) and functioning of the elbow allow critical positioning of the hand in space and the performance of ADLs. The physiologic full ROM of the elbow is $0^{\circ}$ of extension to $140^{\circ}-150^{\circ}$ of flexion, $80^{\circ}-85^{\circ}$ of supination, $75^{\circ}-80^{\circ}$ of pronation [3]. A functional ROM for ADLs has been shown to be $100^{\circ}$ for both the flexion-extension $\operatorname{arc}\left(30^{\circ}-130^{\circ}\right)$ and the pronation-supination $\operatorname{arc}\left(50^{\circ}\right.$ in either direction) [4]. However, this suggested functional ROM has been recently brought into question, as contemporary ADLs, such as those associated with cell phone use and computer tasks, may require increased ROM [5-7]. Loss of ROM in the elbow joint has been defined as loss of extension greater than $30^{\circ}$ or flexion of less than $120^{\circ}$, though it is important to note functional limitations can be appreciated with a less severe ROM loss [8]. Due to the debilitating nature of elbow contracture, the goals of treatment include restoring a more functional arc of motion, decreasing or eliminating pain, and providing joint stability [9]. While some patients can be successfully managed with nonoperative treatment, such as physical therapy, which may or may not include the use of dynamic or static splinting, some cases are refractory to conservative treatment and surgery is indicated [9].

Both open and arthroscopic surgical contracture release techniques have demonstrated the ability to restore motion to a stiff elbow [1-2,8-16], however, the optimal postoperative rehabilitation protocol following surgical contracture release remains unclear [10]. Some authors recommend continuous passive motion (CPM) after surgical contracture release [11-15] to begin elbow ROM immediately postoperatively, but there is limited scientific evidence to support the use of CPM $[14,16]$. There are also disadvantages of CPM use to consider, including the bulky nature of the CPM device and increased costs to the patient.

The purpose of this study is to evaluate the long-term functional results of elbow contracture release surgery, in addition to the effect of occupational therapy (OT) or physical therapy (PT)-guided self-directed rehabilitation program following surgery, without the use of continuous passive motion (CPM) devices. We hypothesize that patients maintain improvement in ROM when using this non-CPM rehabilitation approach. 


\section{Materials And Methods}

This study's protocol was approved by the Institutional Review Board (IRB \# PRAMS 039744EP). The orthopedic surgical database at our institution was queried for patients who had undergone elbow contracture release surgery, open or arthroscopic, by the senior author, from June 2005 to December 2016. This was a single-center study. Patients who met eligibility requirements were contacted by the research associate via telephone. Inclusion criteria were skeletally mature subjects 18 years of age or older who underwent open or arthroscopic contracture release performed by the senior author between June 1, 2005, and March 30, 2017. Exclusion criteria were less than 18 years old and inability to provide informed consent. Informed consent was obtained, and a research-only visit was scheduled with the research associates.

The research visit evaluation was conducted by the research associate and medical student for all patients and included objective measurements of ROM, strength, and neurological sensory testing. ROM measurements included: elbow flexion, extension, pronation, and supination using standard universal goniometer measurements. Grip strength was measured using a hand dynamometer. Other physical examinations included an assessment of Tinel's sign at the elbow, cubital tunnel stretch test, ulnar nerve subluxation, and the 2-point discrimination test [17-21]. Threshold sensitivity testing included the SemmesWeinstein monofilament test [22]. Each patient completed a Hand Pain and Numbness Diagram [23] (Appendix A). Subjective outcome measures were then completed by the patient, including American Shoulder and Elbow Surgeons-elbow (ASES-e) [24], Simple Shoulder Test- elbow (SST-e) [25], Disabilities of the Arm and Shoulder (DASH) [26], Mayo Elbow Performance Index (MEPI) [27], Short Form-36 (SF-36) [28], as well as an investigator-written questionnaire (Appendix B). The ASES-e was scored by subscale (pain, function, and surgery satisfaction), with the ASES pain score ranging from $0-50$ points, with 50 points indicating no pain; function score ranging from $0-36$ points, with 36 points being full functionality; and surgery satisfaction ranging from $0-10$ points, 10 being full satisfaction. The SST-e clinical outcome score assessed the patient's ability to perform ADLs and was scored on a scale of $0-100$, with 0 being unable to perform and 100 being able to easily perform, The DASH assessed functionality, pain, and other symptoms and was scored on a scale of $0-100$, with 0 equating no disability and 100 being most severe disability. The MEPI measured elbow function across four domains: pain, stability, motion, and daily functional tasks and was scored on a scale of 0-100. MEPI scores are categorized as excellent (90-100), good (75-89), fair (60-74), and poor (0-59). The SF 36 score reflects the physical function score and was scaled 0-100, with 100 being a greater health-related quality of life.

The postoperative rehabilitation protocol was standardized for all 19 patients. In the immediate postoperative period, the patient was immobilized in a padded splint with their arm in full extension and elevated overnight via the intravenous (IV) pole. Patients who had an outpatient arthroscopic procedure were still splinted in full extension and asked to keep their arm elevated on pillows overnight. The splint was removed on postoperative Day 1 and active-assisted ROM in flexion, extension, pronation, and supination were started under instruction by occupational therapy. For inpatients, the patients were instructed to continue with their ROM exercises every waking hour on hour until they were able to see their outpatient therapist. For outpatients, they were scheduled to see the therapist the following day after surgery for splint removal and to start exercises. Patients were also provided with a nighttime extension splint. A sling was provided for resting comfort only during the day and patients were advised to wean from sling use by two weeks postoperative or based on their comfort level. Patients attended regular sessions for occupational or physical therapy to continue with stretching. Patients were provided a daily home exercise program (HEP) with emphasis on the terminal end ROM by OT/PT and were advised to complete the HEP multiple times daily. Patients continued with their OT/PT session and HEP until independence with ADLs was achieved and full ROM was achieved or ROM progress had plateaued.

Data are represented as mean \pm standard deviation (SD) for each group and a comparison of the groups was done using the paired t-test (two-tailed). Statistical analysis of the data was performed using GraphPad Prism8 (GraphPad Software, San Diego, CA).

\section{Results}

We were able to enroll 19 patients in the study: six female and 13 male. The mean age of the study population at the time of elbow contracture release surgery was 48.6 ( $\mathrm{SD} \pm 13.2$, range 19-71). Time from surgery to final clinical postoperative follow-up averaged 8.1 months ( $\mathrm{SD} \pm 6.3$, range $2-21$ ). The average time of follow-up from surgical date to research visit was 58.9 months ( $\mathrm{SD}^{ \pm} 39.8$, range 22-117). Further demographic and descriptive data are reported in Table 1. 


\section{Cureus}

\begin{tabular}{ll} 
Characteristic & Mean \pm SD or Number of Patients (\%) \\
Age & $(\mathrm{n}=19)$ \\
Sex & $48.6 \pm 13.2$ \\
Male & $13(68.4)$ \\
Female & $6(31.6)$ \\
Laterality & \\
Left & $9(47.4)$ \\
Right & $10(52.6)$ \\
Handedness & \\
Dominant & $9(47.4)$ \\
Non-Dominant & $10(52.6)$ \\
Heterotopic Ossification & $6(31.6)$ \\
Prior Surgery for Initial Injury & $11(57.9)$ \\
Surgical Approach & $12(63.2)$ \\
Open & $15(79.9)$ \\
Arthroscopic & $4(21.1)$ \\
Ulnar Nerve Transposed & \\
\hline
\end{tabular}

TABLE 1: Patient characteristics

Of the 19 patients, 15 underwent an open contracture release and four an arthroscopic contracture release. Operative reports provided details about the surgical approach and exposure for both open and arthroscopic contracture release procedures. Of the 15 open elbow contracture release surgeries, 14 were done through a posterior midline incision, the other through a medial incision. Regarding the deep exposure for surgical release, three were performed via the medial approach only, four via the lateral approach only, and eight via both the medial and lateral approaches. Twelve patients had an ulnar nerve transposition at the time of elbow contracture surgery, with one being a revision. Indication for transposition included scarring, compression, or mobilization of a nerve to allow for the release of the posterior bundle of the medial collateral ligament to increase ROM. Table 2 further details the surgical approach for each of the 15 patients who underwent an open contracture release. 


\section{Cureus}

\begin{tabular}{|c|c|c|c|c|c|c|}
\hline & Incision & $\begin{array}{l}\text { Medial } \\
\text { approach }\end{array}$ & $\begin{array}{l}\text { Lateral } \\
\text { approach }\end{array}$ & $\begin{array}{l}\text { Ulnar nerve } \\
\text { transposition }\end{array}$ & $\begin{array}{l}\text { Heterotopic } \\
\text { bone } \\
\text { excision }\end{array}$ & Other \\
\hline 1 & Posterior & $\mathrm{x}$ & $x$ & $x$ & $\mathrm{x}$ & \\
\hline 2 & Posterior & $\mathrm{x}$ & & $\mathrm{x}$ & $\mathrm{x}$ & \\
\hline 3 & Posterior & $\mathrm{x}$ & $\mathrm{x}$ & $\mathrm{x}$ & $\mathrm{x}$ & Removal of hardware \\
\hline 4 & Posterior & $\mathrm{x}$ & $\mathrm{x}$ & $x$ & $\mathrm{x}$ & \\
\hline 5 & Posterior & $\mathrm{x}$ & $\mathrm{x}$ & $\mathbf{x}$ & & Removal of loose bodies \\
\hline 6 & Posterior & & $x$ & & & LCL repair; radial head replacement \\
\hline 7 & Medial & $\mathrm{x}$ & $x$ & $x$ & & $\begin{array}{l}\text { Removal of hardware; radial head replacement; } L C L \\
\text { reconstruction; } M C L \text { reconstruction; coronoid reconstruction }\end{array}$ \\
\hline 8 & Posterior & $\mathrm{x}$ & $\mathrm{x}$ & $\mathrm{x}$ & $x$ & \\
\hline 9 & Posterior & & $x$ & & $\mathrm{x}$ & Removal of hardware \\
\hline 10 & Posterior & $\mathrm{x}$ & $\mathrm{x}$ & $\mathrm{x}$ & $\mathrm{x}$ & Radial nerve release; triceps repair \\
\hline 11 & Posterior & $\mathrm{x}$ & & $\mathbf{x}^{\star}$ & & \\
\hline 12 & Posterior & $\mathrm{x}$ & & $\mathrm{x}$ & & Removal of loose bodies \\
\hline 13 & Posterior & & $\mathrm{x}$ & $\mathrm{x}$ & & Removal of hardware \\
\hline 14 & Posterior & $\mathrm{x}$ & $x$ & $x$ & & ORIF capitellar trochlear distal humerus malunion; LCL repair \\
\hline 15 & Posterior & & $x$ & & & $\begin{array}{l}\text { Revision ORIF ulnar segmental fracture; open reduction } \\
\text { radial capitellar joint; LCL repair }\end{array}$ \\
\hline
\end{tabular}

TABLE 2: Elbow contracture release open surgical approach

*revision ulnar nerve transposition performed by a different surgeon

Lateral Collateral Ligament (LCL); Medial Collateral Ligament (MCL); Open Reduction Internal Fixation (ORIF)

The mechanisms of initial injury resulting in elbow contracture for all 19 patients included non-traumatic (osteoarthritis) and post-traumatic (motor vehicle accidents, falls, bicycling accidents) mechanisms.

The results comparing preoperative ROM to research visit ROM for the 19 patients are summarized in Table 3.

\begin{tabular}{|l|llllllll|}
\hline & \multicolumn{7}{l}{ Preoperative ROM } & \multicolumn{7}{ll}{ Research visit ROM } \\
& Average & SD & Range & Average & SD & Range & $p$ Value \\
\hline Flexion & 97.6 & 32.3 & 125 & 131.3 & 14.4 & 65 & 0.0003 \\
Extension & 27.1 & 22.0 & 85 & 24.7 & 22.7 & 75 & 0.6886 \\
Flexion-extension arc & 72.1 & 42.4 & 115 & 106.6 & 30.1 & 140 & 0.0022 \\
Pronation & 71.8 & 24.6 & 90 & 73.2 & 14.5 & 50 & 0.7720 \\
Supination & 60.0 & 35.0 & 90 & 68.2 & 24.2 & 90 & 0.2271 \\
Pronation-supination arc & 131.8 & 53.2 & 180 & 141.3 & 33.7 & 135 & 0.3306 \\
\hline
\end{tabular}

TABLE 3: Preoperative ROM vs. research visit ROM

Range of Motion (ROM)

The difference between preoperative flexion and research visit flexion measurements was statistically significant $(\mathrm{p}<0.001)$, as was the difference between preoperative flexion-extension arc and research visit flexion-extension arc $(\mathrm{p}<0.01)$. There was no significant difference between preop extension and research visit extension measurements $(\mathrm{p}<0.6886)$ and no significant differences between preoperative and research visit pronation $(\mathrm{p}=0.772)$, supination $(\mathrm{p}=0.2271)$, and the pronation-supination $\operatorname{arc}(\mathrm{p}=0.3306)$. The mean change in the flexion-extension arc between the preoperative visit and the research visit was $36.4^{\circ}$ (SD \pm 39.7 , range: min. $-15^{\circ}$, max. $110^{\circ}$ ), and the mean change in the pronation-supination arc was $9.0^{\circ}$ (SD \pm 42.41 , range: $\min .-45^{\circ}$, max. $130^{\circ}$ ). The flexion-extension arc at preoperative, immediate postoperative, final postoperative clinic visit, and research visit time points for all 19 patients is individually plotted in Figure 1. 


\section{Cureus}

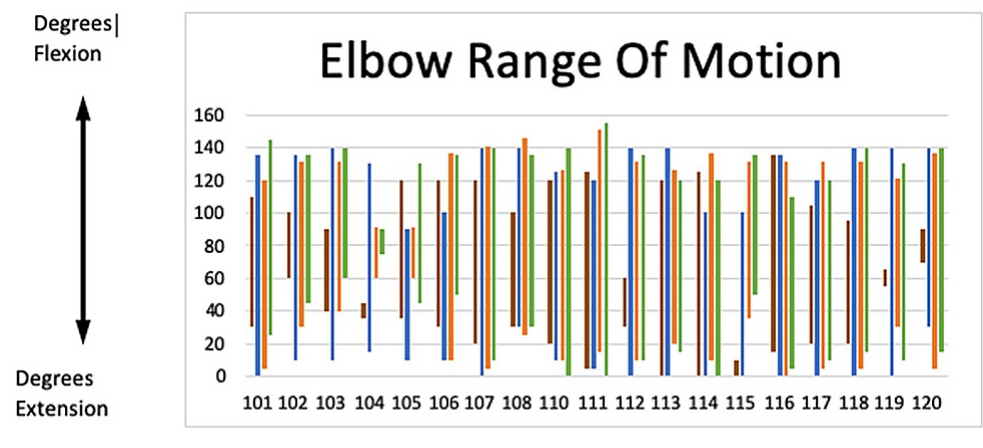

Subject ID

\section{FIGURE 1: Elbow range of motion}

Red: Preoperative ROM; Blue: Immediate postoperative ROM (obtained from the operative report or first postoperative clinic visit when data are not available in the operative report); Orange: Final clinic visit ROM; Green: Research visit ROM

Range of Motion (ROM)

The grip strength mean of the operative side was $30.0 \mathrm{lbs}$. ( $\mathrm{SD} \pm 22.3$ ) and the grip strength mean of the nonoperative side was $35.8 \mathrm{lbs}$. ( $\mathrm{SD} \pm 17.6)$. The difference between the two means was not significant $(\mathrm{p}=$ $0.18)$.

All patients completed the following clinical outcome questionnaires to assess pain, function, and surgery satisfaction: ASES-e, SST-e, DASH, MEPI, and SF-36. The average scores are reported in Table 4.

\begin{tabular}{|c|c|c|c|}
\hline & Average & SD & Range \\
\hline ASES-e pain score & 38.00 & 12.33 & $4-50$ \\
\hline ASES-e function score & 31.95 & 3.56 & $24-36$ \\
\hline ASES-e surgery satisfaction score & 8.84 & 1.63 & 5-10 \\
\hline SST-e score & 78.95 & 27.50 & $8.3-100$ \\
\hline DASH score & 15.57 & 19.36 & $0-73.3$ \\
\hline MEPI score & 86.32 & 17.16 & $40-100$ \\
\hline SF 36 score & 73.95 & 24.79 & $15-100$ \\
\hline
\end{tabular}

TABLE 4: Patient questionnaire scores at research visit

American Shoulder and Elbow Surgeons-elbow (ASES-e); Simple Shoulder Test-elbow (SST-e); Disabilities of the Arm and Shoulder (DASH); Mayo Elbow Performance Index (MEPI); Short Form-36 (SF-36)

Each patient completed the Hand Pain and Numbness Diagram (Appendix A). Five patients had ulnar nerve symptoms of pain, paresthesia, and/or decreased sensation. Of these five patients, four had ulnar nerve transposition at the time of elbow contracture release surgery. A total of 12 of the 19 patients underwent an ulnar nerve transposition. The remainder of the physical exam sensory findings are reported in Table 5 . 


\section{Cureus}

\begin{tabular}{|c|c|c|}
\hline & Operative side & Nonoperative side \\
\hline \multicolumn{3}{|l|}{ Tinel's sign } \\
\hline Positive & 5 & 2 \\
\hline Negative & 14 & 17 \\
\hline \multicolumn{3}{|c|}{ Cubital Tunnel Stretch test } \\
\hline Positive & 7 & 4 \\
\hline Negative & 12 & 15 \\
\hline \multicolumn{3}{|c|}{ Ulnar nerve subluxation } \\
\hline Positive & 0 & 0 \\
\hline Negative & 19 & 19 \\
\hline \multicolumn{3}{|c|}{ 2-point discrimination test } \\
\hline \multicolumn{3}{|l|}{ Index } \\
\hline Normal (0-5mm) & 17 & 17 \\
\hline Fair (6-10mm) & 1 & 2 \\
\hline Poor (11-15mm) & 1 & 0 \\
\hline \multicolumn{3}{|l|}{ Small } \\
\hline Normal (0-5mm) & 14 & 17 \\
\hline Fair (6-10mm) & 4 & 2 \\
\hline Poor (11-15mm) & 1 & 0 \\
\hline \multicolumn{3}{|l|}{ Monofilament test ${ }^{\star}$} \\
\hline \multicolumn{3}{|c|}{ Median Nerve Volar Thumb } \\
\hline Normal (2.83) & 14 & \\
\hline Abnormal (> 2.83) & 5 & \\
\hline \multicolumn{3}{|c|}{ Median Nerve Distal Index } \\
\hline Normal (2.83) & 13 & \\
\hline Abnormal (>2.83) & 6 & \\
\hline \multicolumn{3}{|c|}{ Median Nerve Proximal Index } \\
\hline Normal $(2.83)$ & 17 & \\
\hline Abnormal (>2.83) & 2 & \\
\hline \multicolumn{3}{|c|}{ Ulnar Nerve Distal Small } \\
\hline Normal (2.83) & 13 & \\
\hline Abnormal (> 2.83) & 6 & \\
\hline \multicolumn{3}{|c|}{ Ulnar Nerve Proximal Small } \\
\hline Normal (2.83) & 11 & \\
\hline Abnormal (>2.83) & 8 & \\
\hline \multicolumn{3}{|l|}{ Ulnar Nerve Palm } \\
\hline Normal (2.83) & 12 & \\
\hline Abnormal (>2.83) & 7 & \\
\hline
\end{tabular}

TABLE 5: Research visit physical exam sensory measurements

*The monofilament test was divided into normal (2.83 monofilament size) and abnormal (>2.83 monofilament size).

Of the 19 patients enrolled, 16 completed the additional investigator questionnaire (Appendix B). All 16 patients replied yes to the question "Are you happy with the decision to have the contracture release?" and "If given the choice, would you have the surgery again?" Regarding "what patients reported liking most about the post-surgical outcome," eight patients stated increased ROM, eight patients stated reduced pain, and five mentioned better ability to perform ADLs. Patients listed the scar, post-traumatic arthritis, and inability to lift/push heavy objects as things they least like regarding postsurgical outcomes. All 16 patients stated they are able to complete all ADLs without difficulty. Fifteen patients felt their elbow function is better than the day before surgery, one patient felt it was worse. Fourteen patients reported having pain in 
addition to decreased ROM prior to surgery and all fourteen reported their level of pain was reduced after surgery. Of those 14 patients, six patients reported $100 \%$ pain reduction, five patients reported $50 \%-90 \%$ pain reduction, two patients reported $25 \%-50 \%$ pain reduction, and one patient reported $0 \%-25 \%$ pain reduction.

\section{Discussion}

Prior studies have reported satisfactory outcomes following surgical elbow contracture release [11-14,2930]. However, few studies assess long-term outcomes and patient-reported satisfaction. The primary goals of treatment for a stiff elbow are to restore a functional arc of motion, decrease or eliminate pain, and provide stability [9]. In the current study, we found maintenance in flexion and the flexion-extension arc with long-term follow-up, averaging 58.9 months ( $\mathrm{SD}^{ \pm} 39.8$, range 22-117) at the research visit. Our data would support that a patient self-guided postoperative therapy protocol with an outpatient therapy program was effective in maintaining range of motion. Our clinical outcome data showed most patients are able to comfortably complete ADLs, have minimal pain, and are highly satisfied with their long-term functional outcome of surgery and the decision to have elbow contracture release surgery. Our patient population reported excellent long-term outcomes regarding a decrease in pain. The average scores for the patientreported outcome questionnaires demonstrate decreased pain and high satisfaction in regard to surgical outcomes and the responses to the investigator-written questionnaire supported increased ROM, ability to perform ADLs, and decreased pain for the majority of patients enrolled in this study.

In regard to restoring motion, Sojbjerg suggested that elbow flexion is more important than extension in order to perform ADLs [8]. More recently, increased functional ROM degrees have been suggested to adequately complete contemporary activities of daily living, as holding a cell phone to the ear may require increased flexion, and typing on a computer requires increased pronation [3,5-7]. Our clinical outcome data would support that elbow contracture release surgery maintains increased ROM arc and flexion, decreased pain, and functionality long-term. The SST clinical outcome score assesses the patient's ability to perform ADLs such as performing work duties, dressing, bathing, lifting, carrying, and throwing, with a score of 0 equating to unable to perform and 100 being able to easily perform ADLs. Our cohort's average SST score was 78.95 , indicating that most patients are able to successfully perform ADLs. The DASH clinical outcome scores were utilized to assess the degree of difficulty in performing differing physical activities with the affected arm, the severity of pain symptoms, activity-related pain, tingling, weakness, and stiffness, as well as the affected elbow's impact on social activities, work, sleep, and self-image. Our cohort's average DASH score of 15.57 is rather low, indicating close to no disability when using their surgically released elbow. The MEPI measures elbow function across four domains: pain, stability, motion, and daily functional tasks. Our cohort's average MEPI score of 86.32 is considered a "good" score categorization. This patient cohort consistently demonstrated favorable clinical outcome scores in terms of ability, pain, and satisfaction. In doing so, we are able to counsel patients that they are likely to return to full ability and pain-free ADLs following elbow contracture release surgery with sustained improvement in their elbow flexion.

There are reports of the use of CPM devices in the postoperative period following surgical elbow contracture release [11-15]. Some studies have suggested CPM use following surgical elbow contracture release demonstrates advantageous effects in restoring flexion and extension ROM when compared to no-CPM use and extension splinting [14]. A retrospective study by Aldridge et al. reported an improvement in the total arc of motion of $45^{\circ} \pm 3^{\circ}$ in patients treated with CPM compared to $26^{\circ} \pm 5^{\circ}$ in those not treated postoperatively with CPM. Gates et al. demonstrated a significant difference in the increased mean total arc of motion between the CPM cohort and the non-CPM cohort of $27^{\circ}$ and $48^{\circ}$, respectively [14]. However, an important limitation of this study by Gates et al. is that there was no standardization between the CPM and no-CPM cohort, as prior to contracture release the no-CPM cohort had substantially stiffer elbows than the CPM cohort [14]. Cohen and Hastings reported a mean arc of motion increase from $74^{\circ}$ to $129^{\circ}$ in 22 patients following surgical elbow contracture release and postoperative CPM use [13]. In contrast, Lindenhovius et al. demonstrated that patients, evaluated between seven months and 56 months postoperatively, who did not use CPM in their postoperative rehabilitation protocol had similar results in terms of ROM change and restoration when compared to the literature of patients who did use CPM [16]. The improvement in ROM measurements in our study support sustained ROM in the flexion-extension arc at the long-term follow-up, ranging from 22 months to 58 months, without the use of CPM. Our study demonstrates a statistically significant improvement in flexion ROM, leading the authors to suspect that extension ROM may be limited over time due to the anterior capsule of the elbow joint getting tight over time and likely scarring down to the anterior humerus, as most elbow resting positions in some degree of flexion and terminal extension may therefore be lost. Ultimately, our study demonstrates patients do well long-term with a self-directed/home therapy program guided by OT/PT without the use of CPM.

Given the nature of the ulnar nerve's close anatomical proximity and involvement in elbow contracture release, it is important to assess residual nerve dysfunction at the long-term follow-up. On physical exam sensory testing, most patients in our cohort reported minimal sensory dysfunction (Table 5). These findings were also supported by our cohort's low DASH score average (15.57). The Hand Pain and Numbness Diagram also demonstrated five patients to have sensory symptoms in the ulnar nerve distribution, and four of these patients did have an ulnar nerve transposition at the time of elbow contracture release surgery. This is important when counseling patients regarding residual nerve dysfunction and symptoms when the ulnar nerve is transposed. However, we had very limited preoperative sensory testing data to appropriately determine if sensory function improved, worsened, or stayed the same following surgery. Strength in the affected arm at long-term follow-up compared favorably to the unaffected arm. The grip strength mean of the operative side was $30.05 \mathrm{lbs}$. ( $\mathrm{SD} \pm 22.3$ ) and the grip strength mean of the non-operative side was 35.8 lbs. (SD \pm 17.6 ). While we cannot comment on the maintenance of strength from surgery to long-term follow-up, we can counsel patients that their grip strength in the surgical hand will return to similar to the non-operative side.

We recognize this study has several limitations. This is a case series and, therefore, a small sample size. Second, while this study reported the long-term outcome of surgical elbow contracture release in a patient group, we did not have a comparison control group to demonstrate the direct effects of the use of CPM versus no CPM and, therefore, future research should focus on the direct comparison between these rehabilitation protocols. Third, preoperative ROM measurements were gathered via retrospective chart review, which leaves room for the introduction of bias and human error, however, a goniometer is routinely used in the clinic to measure ROM. Lastly, we recognize the test measurements performed at a 


\section{Cureus}

comprehensive research visit were not also available in the preoperative setting for our patient cohort due to the study's design and the overall study design and methods can be improved upon in the future.

\section{Conclusions}

Elbow contracture release surgery demonstrates improvement and maintenance in flexion and flexionextension arc with long-term follow-up. The long-term postoperative outcome for surgical elbow contracture release followed by a self-directed, OT/PT-guided physical therapy program without CPM is favorable. Furthermore, patients were satisfied with long-term postoperative outcomes related to performing ADLs, decreased pain, and the decision to have the surgery.

\section{Appendices}

\section{Appendix A}

Hand Pain and Numbness Diagram

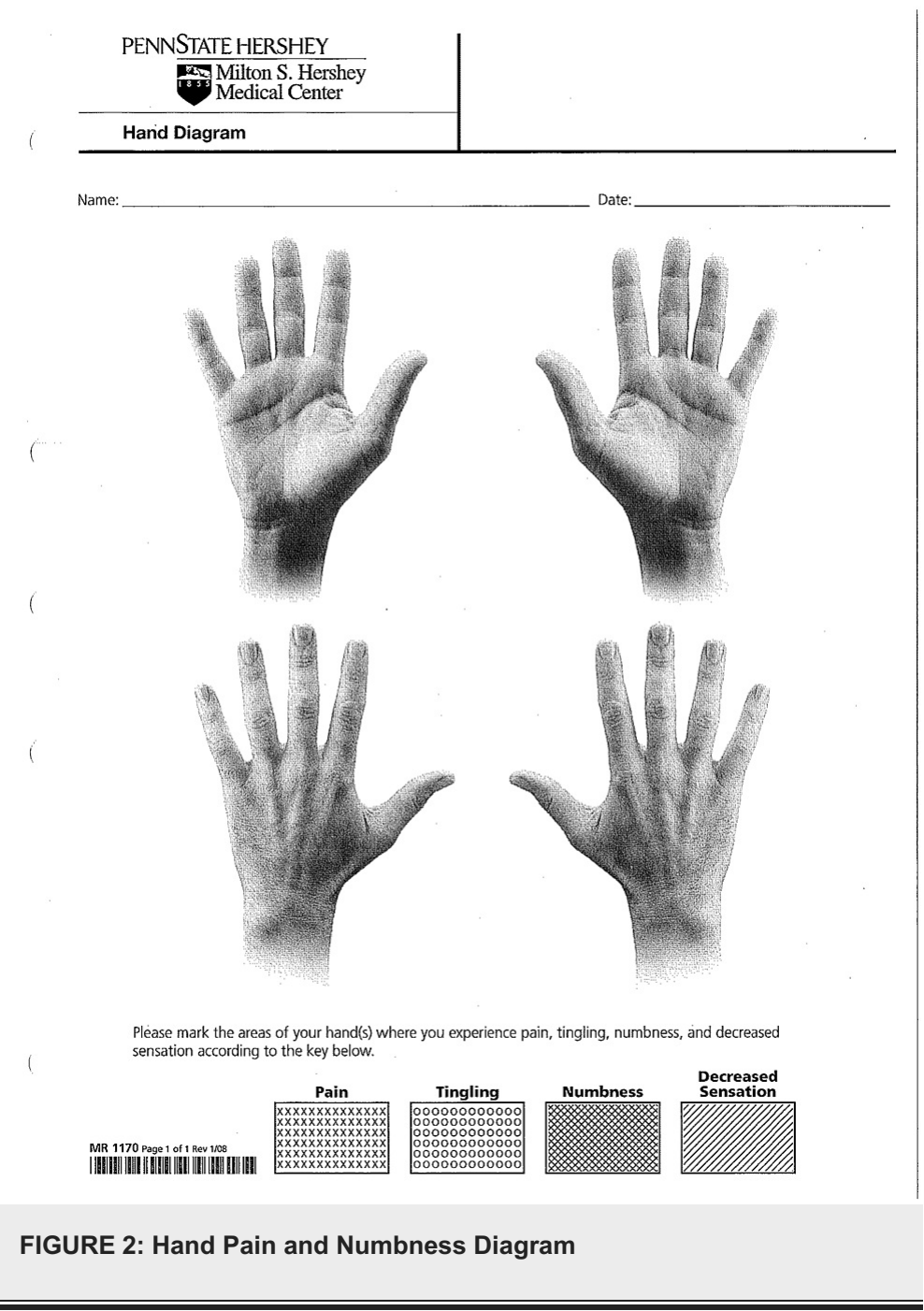

\section{Appendix B}

Investigator Written Questionnaire

Please take the time to read and answer each question carefully.

1.) On the line below, please indicate your level of pain prior to the contracture release of your elbow.

No Pain | Worst Possible Pain

2.) Do you currently have elbow pain? Yes__ No _

a.) If Yes, please indicate your pain level on the line below.

No Pain | | Worst Possible Pain

b.) If No, how long after the contracture release did it take for your elbow pain to go away? 


\title{
Cureus
}

\author{
3.) Are you happy with the decision to have the contracture release? Yes___ No _ \\ 4.) Did anything influence your decision to have the contracture release? Yes___ No \\ a.) If Yes, please explain \\ 5.) If given the choice, would you have the surgery again? Yes \\ No \\ 6.) Have you had any additional surgeries since having the contracture release? Yes
}

a.) If Yes, what was done?

7.) What do you like most as a result of the contracture release?

8.) What do you like least as a result of the contracture release?

9.) What activities of daily living are you still able to do without much difficulty? (ie: brushing teeth, brushing hair, washing dishes, bathing, personal hygiene)

10.) What activities of daily living do you have trouble performing? (ie: brushing teeth, brushing hair, washing dishes, bathing, personal hygiene)

11.) Is your elbow function worse / the same / better than the day before your surgery? Please circle one.

12.) What advice would you give someone considering a contracture release?

13.) After the contracture release, was your level of pain reduced? Yes No

a.) If Yes, please rate how much your pain was reduced by having the contracture release.

Slightly (0-25\%) Moderately (25-50\%) Significantly (50-90\%) No Pain

14.) Please feel free to provide additional comments:

Thank you for completing this survey.

\section{Additional Information \\ Disclosures}

Human subjects: Consent was obtained or waived by all participants in this study. Penn State College of Medicine, Institutional Review Board, Human Subjects Protection Office issued approval PRAMS039744EP. On 6/5/2020, the IRB approved the above-referenced Continuing/Administrative Review. Animal subjects: All authors have confirmed that this study did not involve animal subjects or tissue. Conflicts of interest: In compliance with the ICMJE uniform disclosure form, all authors declare the following: Payment/services info: All authors have declared that no financial support was received from any organization for the submitted work. Financial relationships: April Armstrong declare(s) personal fees from Zimmer Biomet. April Armstrong declare(s) personal fees from Aevumed. April Armstrong declare(s) personal fees from Globus . Other relationships: All authors have declared that there are no other relationships or activities that could appear to have influenced the submitted work.

\section{References}

1. Keschner MT, Paksima N: The stiff elbow. Bull NYU Hosp Jt Dis. 2007, 65:24-8.

2. Adolfsson L: Post-traumatic stiff elbow. EFORT Open Rev. 2018, 3.210-6. 10.1302/2058-5241.3.170062

3. Thompson IC: Arm: physical exam. Netter's Concise Orthopaedic Anatomy. Saunders Elsevier, Philadelphia, Pennsylvania; 2016. 125 .

4. Morrey BF, Askew LJ, Chao EY: A biomechanical study of normal functional elbow motion . J Bone Joint Surg Am. 1981, 63:872-7.

5. Sardelli M, Tashiian RZ, MacWilliams BA: Functional elbow range of motion for contemporary tasks . J Bone Joint Surg Am. 2011, 93:471-7. 10.2106/JBJ.I.01633

6. Magermans DJ, Chadwick EK, Veeger HE, van der Helm FC: Requirements for upper extremity motions during activities of daily living. Clin Biomech (Bristol, Avon). 2005, 20:591-9. 10.1016/..clinbiomech.2005.02.006

7. Oosterwijk AM, Nieuwenhuis MK, van der Schans $\mathrm{CP}$, Mouton LI: Shoulder and elbow range of motion for the performance of activities of daily living: a systematic review. Physiother Theory Pract. 2018, 34:505-28. 10.1080/09593985.2017.1422206

8. Søjbjerg JO: The stiff elbow. Acta Orthop Scand. 1996, 67:626-31. 10.3109/17453679608997771

9. Streubel PN, Cohen MS: Open surgical release for contractures of the elbow. J Am Acad Orthop Surg. 2015, 23:328-38. 10.5435/JAAOS-D-14-00051

10. Lindenhovius AL, Jupiter JB: The posttraumatic stiff elbow: a review of the literature . J Hand Surg Am. 2007, 32:1605-23. 10.1016/j.jhsa.2007.09.015

11. Heirweg S, De Smet L: Operative treatment of elbow stiffness: evaluation and outcome . Acta Orthop Belg. 2003, 69:18-22.

12. Husband JB, Hastings $\mathrm{H}$ 2nd: The lateral approach for operative release of post-traumatic contracture of the 


\section{Cureus}

elbow. J Bone Joint Surg Am. 1990, 72:1353-8.

13. Cohen MS, Hastings $\mathrm{H}$ 2nd: Post-traumatic contracture of the elbow. Operative release using a lateral collateral ligament sparing approach. J Bone Joint Surg Br. 1998, 80:805-12. 10.1302/0301-620x.80b5.8528

14. Gates, H.S., 3rd, F.L. Sullivan, and J.R. Urbaniak: Anterior capsulotomy and continuous passive motion in the treatment of post-traumatic flexion contracture of the elbow. A prospective study. J Bone Joint Surg Am. 1992, 74:1229-34.

15. Morrey BF: Surgical treatment of extraarticular elbow contracture. Clin Orthop Relat Res. 2000, 370:57-64. 10.1097/00003086-200001000-00007

16. Lindenhovius AL, van de Luijtgaarden $\mathrm{K}$, Ring $\mathrm{D}$, Jupiter J: Open elbow contracture release: postoperative management with and without continuous passive motion. J Hand Surg Am. 2009, 34:858-65. 10.1016/j.jhsa.2009.01.003

17. Novak, C.B., Lee GW, Mackinnon SE, Lay L: Provocative testing for cubital tunnel syndrome . J Hand Surg Am. 1994, 19:817-20. 10.1016/0363-5023(94)90193-7

18. Buehler MI, Thayer DT: The elbow flexion test. A clinical test for the cubital tunnel syndrome .Clin Orthop Relat Res. 1988, 213-6.

19. Calfee RP, Manske PR, Gelberman RH, Van Steyn MO, Steffen J, Goldfarb CA: Clinical assessment of the ulnar nerve at the elbow: reliability of instability testing and the association of hypermobility with clinical symptoms. J Bone Joint Surg Am. 2010, 92:2801-8. 10.2106/BJS.J.00097

20. Dellon AL: Review of treatment results for ulnar nerve entrapment at the elbow . J Hand Surg Am. 1989, 14:688-700. 10.1016/0363-5023(89)90192-5

21. Hsu HY, Kuo LC, Chiu HY, Jou IM, Su FC: Functional sensibility assessment. Part II: effects of sensory improvement on precise pinch force modulation after transverse carpal tunnel release. J Orthop Res. 2009, 27:1534-9. 10.1002/jor.20903

22. McGill M, Molyneaux L, Yue DK: Use of the Semmes-Weinstein 5.07/10 gram monofilament: the long and the short of it. Diabet Med. 1998, 15:615-7. 10.1002/(SICI)1096-9136(199807)15:7<615::AIDDIA641>3.0.CO;2-P

23. Katz JN, Stirrat CR, Larson MG, Fossel AH, Eaton HM, Liang MH: A self-administered hand symptom diagram for the diagnosis and epidemiologic study of carpal tunnel syndrome. J Rheumatol. 1990, 17:14958.

24. King GJ, Richards RR, Zuckerman JD, et al.: A standardized method for assessment of elbow function . J Shoulder Elbow Surg. 1999, 4:351-4. 10.1016/s1058-2746(99)90159-3

25. Godfrey J, Hamman R, Lowenstein S, Briggs K, Kocher M: Reliability, validity, and responsiveness of the simple shoulder test: psychometric properties by age and injury type. J Shoulder Elbow Surg. 2007, 16:260-7 10.1016/i.je 2006.07.003

26. Hudak PL, Amadio PC, Bombardier C: Development of an upper extremity outcome measure: the DASH (disabilities of the arm, shoulder and hand) [corrected]. The Upper Extremity Collaborative Group (UECG) Am J Ind Med. 1996, 29:602-8. 10.1002/(SICI)1097-0274(199606)29:6<602::AID-AJIM4>3.0.CO;2-L

27. Morrey BF, An KN, Chao EY: Functional evaluation of the elbow. The Elbow and Its Disorders. 2nd ed. Saunders Elsevier, Philadelphia, PA; 1993. 66-73.

28. Bunevicius A: Reliability and validity of the SF-36 Health Survey Questionnaire in patients with brain tumors: a cross-sectional study. Health Qual Life Outcomes. 2017, 15:92. 10.1186/s12955-017-0665-1

29. Tan V, Daluiski A, Simic P, Hotchkiss RN: Outcome of open release for post-traumatic elbow stiffness . J Trauma. 2006, 61:673-8. 10.1097/01.ta.0000196000.96056.51

30. Park MJ, Kim HG, Lee JY: Surgical treatment of post-traumatic stiffness of the elbow . J Bone Joint Surg Br. 2004, 86:1158-62. 10.1302/0301-620x.86b8.14962 